\title{
THE EFFECT OF TEMPERATURE ON IN VITRO DIGESTIBILITY, FAT GLOBULAR SIZE AND FREE FATTY ACID BIO-AVAILABILITY IN MILK FAT
}

\author{
Michał Smoczyński $\bowtie$ \\ Department of Dairy Science and Quality Management, University of Warmia and Mazury in Olsztyn \\ Oczapowskiego 7, 10-719 Olsztyn, Poland
}

\begin{abstract}
Background. The rate at which a particular fat is digested has an effect on its bioavailability and on the lipid profile of the blood. Milk fat is a very complex mixture of triacylglycerols, resulting in a very wide melting range (from -40 to $+40^{\circ} \mathrm{C}$ ). Because temperature has a pronounced effect on the physicochemical state of milk fat (i.e. crystallisation of different fat fractions), this study analysed the bioavailability of milk fat at different temperatures.

Material and methods. A simplified model simulating digestion in the intestine at various temperatures was used. The released fatty acids and the changes in the emulsion of milk fat under the effect of lipase were compared.

Results. The amount and profiles of the released fatty acids varied depending on the incubation temperature of the studied sample. At lower temperatures, the fractions which were hydrolysed to a greater degree were those which contained more unsaturated oleic acid, but contained less C14, C16 and C18 saturated acids. Changes in the emulsion system also differed depending on temperature.

Conclusions. The obtained results indicate that, depending on the temperature, not only different amounts, but also different fractions of milk fat were hydrolysed by lipase, which indicates the role of the physicochemical state of milk fat during its digestion.
\end{abstract}

Keywords: milk fat, fatty acid composition, lipase, fat digestion

\section{INTRODUCTION}

Cow's milk is a significant source of nutrients in the human diet, including fat. Milk lipids occur in an emulsified form, as fat globules with sizes from 0.2 to $15 \mu \mathrm{m}$. Inside the globules, there are mainly nonpolar triacylglycerols surrounded by a so-called milk fat globule membrane (MFGM) around 10-20 nm thick. The membrane is a triple layer of phospholipids (composed of phospho- and sphingolipids constituting $0.2-2 \mathrm{wt} \%$ of milk lipids), also containing proteins, cholesterol, partial glycerides, free fatty acids and other components (Mather, 2000). The main lipid fraction are triacylglycerols, constituting $97-98 \%$ of milk lipids (Bitman and Wood, 1990). In milk fat, over 400 different fatty acids have been identified, but only 15 types of fatty acids are present in an amount greater than $1 \%$ of the total amount of milk fat (Parodi, 2004). Such a wide range of fatty acids results in the formation of a very complex mixture of fractions of triacylglycerols (TAGs), which makes milk fat the most complex fat to occur in nature. TAGs are synthesised 
in the mammary gland, with the participation of enzymes, which results in a particular stereospecificity regarding their position in glycerol. Short-chain acids $(4: 0,6: 0)$ are esterified mainly in position $s n-3$, whereas medium-chain acids $(8: 0-16: 0)$ are mainly present in positions $s n-1$ and $s n-2$. Stearic acid is present mainly in position $s n-1$, while oleic acid is present in positions $s n-1$ and $s n-3$ (MacGibbon and Taylor, 2006; Parodi, 2004). The digestion of fat in humans is a complex physicochemical and enzymatic process. The high degree of dispersion of lipid compounds in milk enables lipolysis to occur effectively. Additionally, the particular structure of the interface is important for the efficient hydrolysis of milk fat globules by lipase (Berton et al., 2012; Smoczyński and Kiełczewska, 2014). This process starts in the oral cavity, but the main process of fat digestion takes place in the duodenum, under the effect of factors secreted by the biliary vesicle and by the pancreas. The main enzyme responsible for hydrolysis of triacylglycerols at this stage is pancreatic lipase. Because of its specificity to the $s n-1,3$ glycerol position, the enzyme converts TAGs to more polar $s n-2$ monoacylglycerols and free fatty acids. They form mixed micelles with the participation of bile salts and, in this form, can be absorbed by intestinal epithelial cells (Bauer et al., 2005).

Milk fat is a very complex mixture of triacylglycerols, which leads to very wide melting range (from -40 to $+40^{\circ} \mathrm{C}$ ). It has been demonstrated that in emulsion form, milk fat mainly crystallises in the $\alpha$ form and, after some time, it partly converts to the $\beta$ ' form and both of these forms occur simultaneously $\left(8^{\circ} \mathrm{C}\right)$, while anhydrous (bulk) milk fat crystallises first in the $\beta$ ' form and later forms the $\alpha$ form (Lopez et al., 2001).

The rate at which a particular fat is digested and the extent to which it is utilised in the human body have an effect on its bioavailability and on the lipid profile in blood and in other organs (Bonnaire et al., 2008). Thus, the physicochemical state of milk fat and the proportion of the crystalline form in its structure may play a significant role in the digestion of milk fat. Because temperature has a pronounced effect on the physicochemical state of milk fat, in this project, the bioavailability of milk fat was studied at different temperatures. For this purpose, the changes in the emulsion of milk fat under the effect of lipase were compared, in a simplified model simulating digestion in the intestine, at different temperatures.

\section{MATERIAL AND METHODS}

\section{Chemicals and reagents}

Lipase from a porcine pancreas Type II (a crude preparation which contains the lipase-colipase complex as well as protease and amylase activity, Cat No 9001$-62-1$ ) and bile salts (containing a mixture of sodium salts of cholic acid $(\sim 50 \%)$ and deoxycholic acid $(\sim 50 \%)$, Cat No 48305$)$ were obtained from Sigma Aldrich (Saint Louis, Missouri, USA). Distilled water was obtained from a Milli-Q water purification unit (Millipore, Milford, USA). All other chemicals and reagents were of analytical grade.

\section{Material and sample preparation}

The material used for the study was homogenised and pasteurised cream $\left(93-95^{\circ} \mathrm{C}, 40 \mathrm{~s}\right)$ with a fat content of $12 \%$. Before the experiment, $5 \mathrm{~cm}^{3}$ samples of the cream were incubated at $10^{\circ} \mathrm{C}, 20^{\circ} \mathrm{C}$ and $36^{\circ} \mathrm{C}$ for a period of 3 hours to crystallise the triacylglycerol fractions.

\section{Lipase-catalysed digestion of fat globules}

After the initial incubation, fat globules in the analysed samples were digested using a simplified model of intestinal digestion. For this purpose, $5 \mathrm{mg} / \mathrm{ml}$ of bile salts $(\mathrm{w} / \mathrm{v})$ were added to the cream samples, followed by $1.5 \mathrm{mg} / \mathrm{ml}$ of lipase (w/v) and the samples were then incubated with moderate mixing at temperatures of $10^{\circ} \mathrm{C}, 20^{\circ} \mathrm{C}$ and $36^{\circ} \mathrm{C}$ for $2 \mathrm{~h}$. Both the bile salts and the enzymes were dissolved in simulated intestinal fluid (SIF) with a pH of 6.8, containing $6.805 \mathrm{~g}$ of $\mathrm{KH}_{2} \mathrm{PO}_{4}(50 \mathrm{mM})$ and $0.896 \mathrm{~g}$ of $\mathrm{NaOH}(22 \mathrm{mM})$ in 1 litre (United States Pharmacopeia, 2003) before being added to the sample. The contents of the enzyme and the bile salts in SIF were 0.05 and $0.1 \mathrm{~g} / \mathrm{cm}^{3}$ respectively. Lipase activity was determined by measuring the amount of free fatty acids released in the analysed sample using the Dole extracting-titratable method with the Deeth et al. (1975) modification. In this method free fatty acids from a sample were extracted by a mixture of 2-propanol: petroleum ether: $2 \mathrm{M} \mathrm{H}_{2} \mathrm{SO}_{4}$ $(40: 10: 1)$ and the determined amount of the ethereal 
layer was titrated with a $0.005 \mathrm{M}$ methanolic solution of $\mathrm{KOH}$ in the presence of $\alpha$-naphthophthalein as an indicator. The lipase activity was expressed in micromoles of fatty acids liberated from 1 gram of the cream sample.

\section{Measurement of particle sizes}

The changes in mean particle size and particle size distribution of fat globules during the hydrolysis process were determined by light scattering measurement in a Mastersizer apparatus (Malvern, Malvern Instruments Ltd., Worcestershire, UK). Milk samples were diluted in water directly in a measuring cell of the apparatus in order to obtain $10-15 \%$ obscuration. The refraction index for water and milk was 1.33 and 1.46, respectively. The particle size distribution was used to determine the mean particle size $d_{(3.2)}$.

Mean particle size $d_{(3.2)}$ is defined as:

$$
d_{(3.2)}=\sum n_{i} d_{i}^{3} / \sum n_{i} d_{i}^{2}
$$

where $n_{i}$ is the number of droplets of diameter $d_{i}$. The results represent mean values from at least five measurements of each milk sample.

\section{Extraction and separation of lipid classes}

After incubation, fat was extracted from the studied samples using the Hara and Radin (1978) method. For this purpose, a hexane : isopropanol mixture $(3 / 2 \mathrm{v} / \mathrm{v})$ was added to $2 \mathrm{~g}$ of the studied sample of cream and then, after $30 \mathrm{~s}$ of intensive mixing, a water solution of sodium sulphate $\left(1 \mathrm{~g}\right.$ of the salt in $15 \mathrm{~cm}^{3}$ of water) was added. After mixing and phase separation, the upper fraction containing milk lipids was collected, and the solvents were evaporated in a vacuum. The lipids were then subjected to solid-phase extraction, in order to separate the lipids into particular fractions. For this purpose, columns filled with silica (UCT, Cusil 156, Bristol, USA) were used. The extraction was performed according to the method described by Hamilton and Comai (1988). Generally, combinations of hexane and methyltertiarybutylether were used to progressively elute lipid classes from the column. The fractions containing triacylglycerols and partial triacylglycerols were then evaporated in a vacuum and subjected to further determinations using gas chromatography.

\section{Analysis of the fatty acid composition}

The obtained fractions containing triacylglycerols and partial triacylglycerols were subjected to methylation, using the IDF standard method (ISO-IDF, 2002). For this purpose, a $0.1 \mathrm{~cm}^{3}$ of saturated ethanol solution of $\mathrm{KOH}$ was added to the studied sample dissolved in hexane $\left(0.05 \mathrm{~g} \mathrm{fat} / 2.5 \mathrm{~cm}^{3}\right)$ and shaken for $1 \mathrm{~min}$ ute. $0.25 \mathrm{~g}$ of crystalline $\mathrm{NaHSO}_{4}$ was then added after 5 minutes. After mixing, the sample was centrifuged for 3 minutes (1000 rpm) and the upper clear solution was subjected to separation by gas chromatography, using a chromatograph produced by Agilent Technologies $(7890 \mathrm{~A}$ model, Agilent Technologies, Palo Alto, USA) with a flame ionisation detector. For the separations, a CP-Sil 88 capillary column was used (length $100 \mathrm{~m}$, diameter $0.25 \mathrm{~mm}$, film thickness $0.20 \mu \mathrm{m}$; Agilent Technologies, Palo Alto, USA). The separation conditions were as follows: the initial oven temperature: $60^{\circ} \mathrm{C}$ for 1 minute, then the temperature was increased gradually to $180^{\circ} \mathrm{C}\left(5^{\circ} \mathrm{C} / \mathrm{min}\right)$. The temperatures of the injector and of the detector were $225^{\circ} \mathrm{C}$ and $250^{\circ} \mathrm{C}$, respectively. The propellant gas was helium with a flow rate of $0.8 \mathrm{~mL} / \mathrm{min}$. The sample was dosed with a 1:50 split of the sample in a $1 \mu \mathrm{L}$ volume. All experiments were performed in triplicate and the results are presented as mean values \pm standard deviation.

\section{Statistical analysis}

The results are presented as mean values of at least three repetitions with standard deviations. One-way analysis of variance (ANOVA) was applied to analyse the data using Fisher's least significance difference (LSD) test for multiple comparisons at a 95\% confidence level. The data was analysed using Statistica version 13.1 for Windows.

\section{RESULTS}

\section{Hydrolysis of milk fat globules at different temperatures}

The milk fat globules were hydrolysed by lipase in the presence of bile salts at different temperatures. The milk samples at temperatures of $10^{\circ} \mathrm{C}, 20^{\circ} \mathrm{C}$ and $36^{\circ} \mathrm{C}$ differ in crystalline fraction content and may correspond to the milk fat consumed almost directly from the fridge, milk fat at room temperature and 


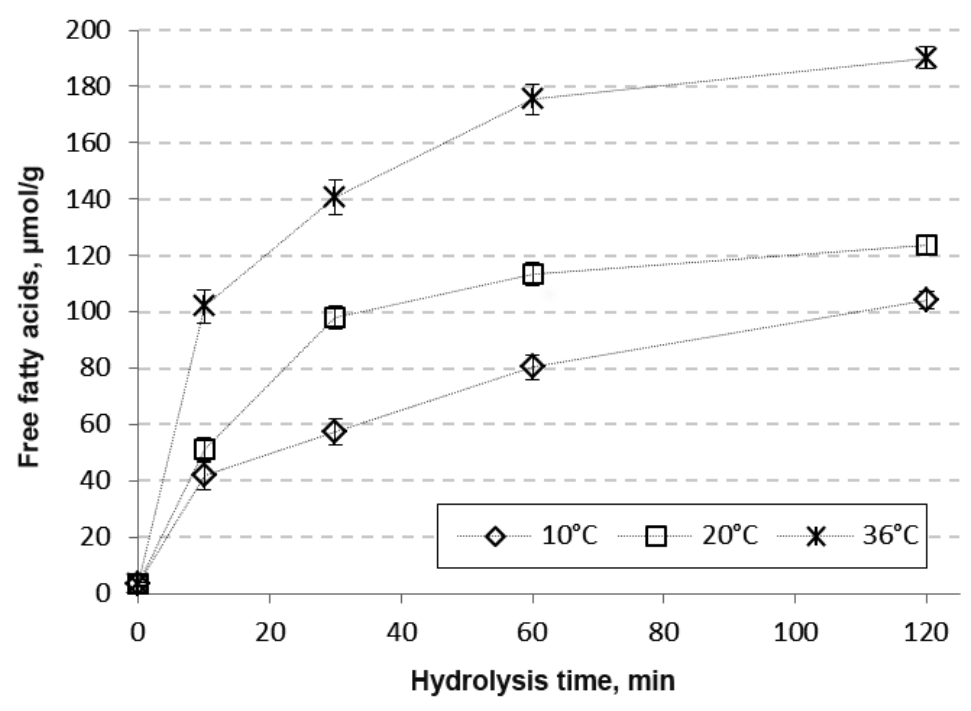

Fig. 1. The amount of fatty acids released from milk fat globules at different temperatures. Each point represents the average of three determinations on separate milk samples \pm standard deviation

physiological temperature respectively, for comparison. The rates of lipid digestion are presented in Figure 1.

The obtained results show that at all three temperatures, the milk fat globules were hydrolysed by lipase, although to a varying extent. The amount of fatty acids released increased with increasing temperature. The highest amount of fatty acids was released at $36^{\circ} \mathrm{C}$ and reached over $190 \mu \mathrm{mol} / \mathrm{g}$ of cream after 2 hours of incubation. At lower temperatures, the amount of free fatty acid was smaller, and there were only slight differences in the levels of released fatty acids at $10^{\circ} \mathrm{C}$ and $20^{\circ} \mathrm{C}$. The amount reached about 104 and $123 \mu \mathrm{mol} / \mathrm{g}$ of cream respectively after two hours of incubation. The difference in the rate of milk fat hydrolysis, like any other chemical reaction, depends on the temperature of incubation, and temperature is the main factor responsible for quantitative differences. With decreasing temperature, more fractions of milk fat crystallise and are less affected by lipase in this form. Although a temperature of $10^{\circ} \mathrm{C}$ differs from physiological conditions, a substantial amount of milk fat was still hydrolysed by lipase in these conditions. The efficiency of lipid hydrolysis may depend to a significant degree on their ability to remove the reaction products from the interface, where the reaction takes place (Blackberg et al., 1981). The physicochemical state of the substrate may also influence the reaction rate. The obtained results indicate that lipase may also be capable of hydrolysing triacylglycerols in crystalline form, which was found in other studies (Bonnaire et al., 2008). The other possible explanation is that inside the milk fat globules, crystalline forms of triacylglycerols separate from other fractions and liquid triacylglycerols are still available for hydrolysis by lipase.

\section{Changes in fat globule size during hydrolysis}

The size of the milk fat globules changed during the process of lipolysis. In this experiment, no dissociating agent was used during the measurements, in order to better resemble natural digestive conditions and not to interfere with the processes of flocculation of milk fat globules. Therefore, the observed results represent complex interactions between fat globules which are present on the surface of homogenised milk fat globules. The particle size distributions obtained are presented in Figure 2.

The changes in particle size during the process of lipolysis are consistent with the results obtained during free fatty acid determination. The changes in the size distribution of the fat globules were similar for the samples of milk incubated at $10^{\circ} \mathrm{C}$ and $20^{\circ} \mathrm{C}$, but were most pronounced at $36^{\circ} \mathrm{C}$. At this temperature, a loss of particles 

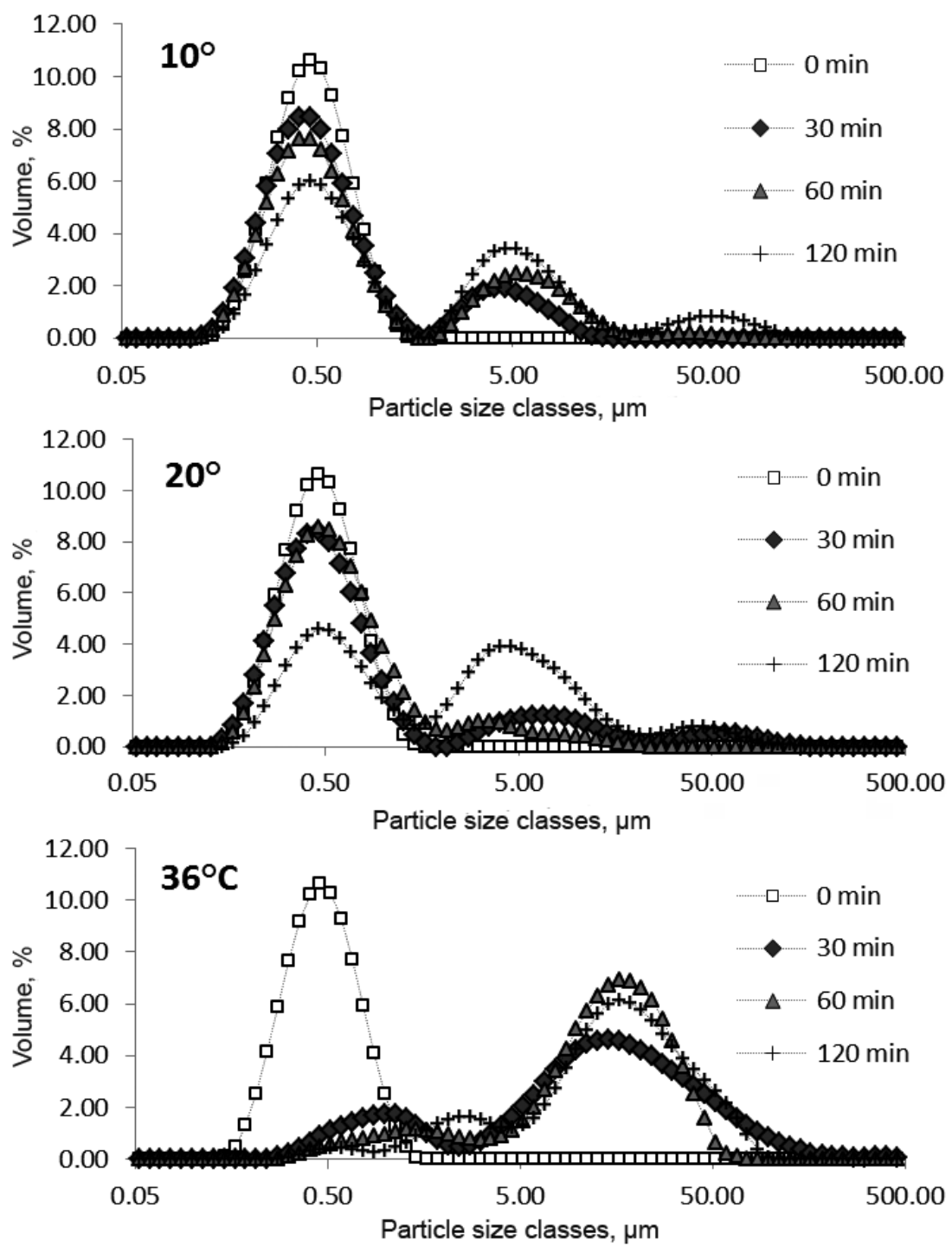

Fig. 2. The changes in particle size of milk fat globules during lipolysis at different temperatures. Each point represents the average of at least five determinations

with small initial sizes can be observed after 30 min of hydrolysis. Simultaneously, larger-sized particles of 5 and $50 \mu \mathrm{m}$ could be observed. The size distribution of fat globules at $36^{\circ} \mathrm{C}$ widened after $30 \mathrm{~min}$ of incubation and more particles of $50 \mu \mathrm{m}$ size appeared. The mean particle diameter $\left(D_{3.2}\right)$ increased from 0.5 to $21.2 \mu \mathrm{m}$ after 90 minutes of hydrolysis at $36^{\circ} \mathrm{C}$, while at $10^{\circ} \mathrm{C}$ and $20^{\circ} \mathrm{C}$ mean particle diameters after 90 minutes were 4.32 and $7.9 \mu \mathrm{m}$ respectively. This could be a result of globule aggregation, flocculation and/or coalescence.
At lower temperatures, particles with small initial sizes did not disappear during the lipolysis process and were still observed after two hours of incubation. The milk fat globule membrane has an inhibitory effect on digestive lipases (Blackberg et al., 1981). This inhibitory effect can be reduced by the cooperation of bile salts and phospholipids with the lipase-colipase system. Bile salts and phospholipids compete and displace the surface active material originally present at the fat-water interface. Lipase-colipase can then bind to the modified 
lipid droplet surface and convert the triacylglycerols into diacylglycerols, monoacylglycerols and free fatty acids. When this process takes place at physiological temperature, most of the triacylglycerol fractions are in a liquid state. It is possible that during modification of the interface, these liquid fractions can "leak" from the inside onto the surface of fat droplets, causing increased flocculation of milk fat globules. The process of flocculation may accelerate lipolysis, because it brings the substrate into closer proximity with the enzyme. In this context, flocculation can be regarded as a desirable phenomenon during the hydrolysis of fat emulsions and may explain the differences in the results of lipolysis at different temperatures observed in this study.

\section{Differences in released fatty acid composition}

Both the stereospecificity connected with the synthesis of triacylglycerols and the stereospecificity of lipase enable efficient absorption of all fatty acids. In this study, the profiles of released fatty acids were compared, depending on the lipolysis temperature.

The diagrams (Fig. 3) present the contents of the main fatty acids in the fractions of partial glycerols and of triacylglycerols after lipolysis. The profile of
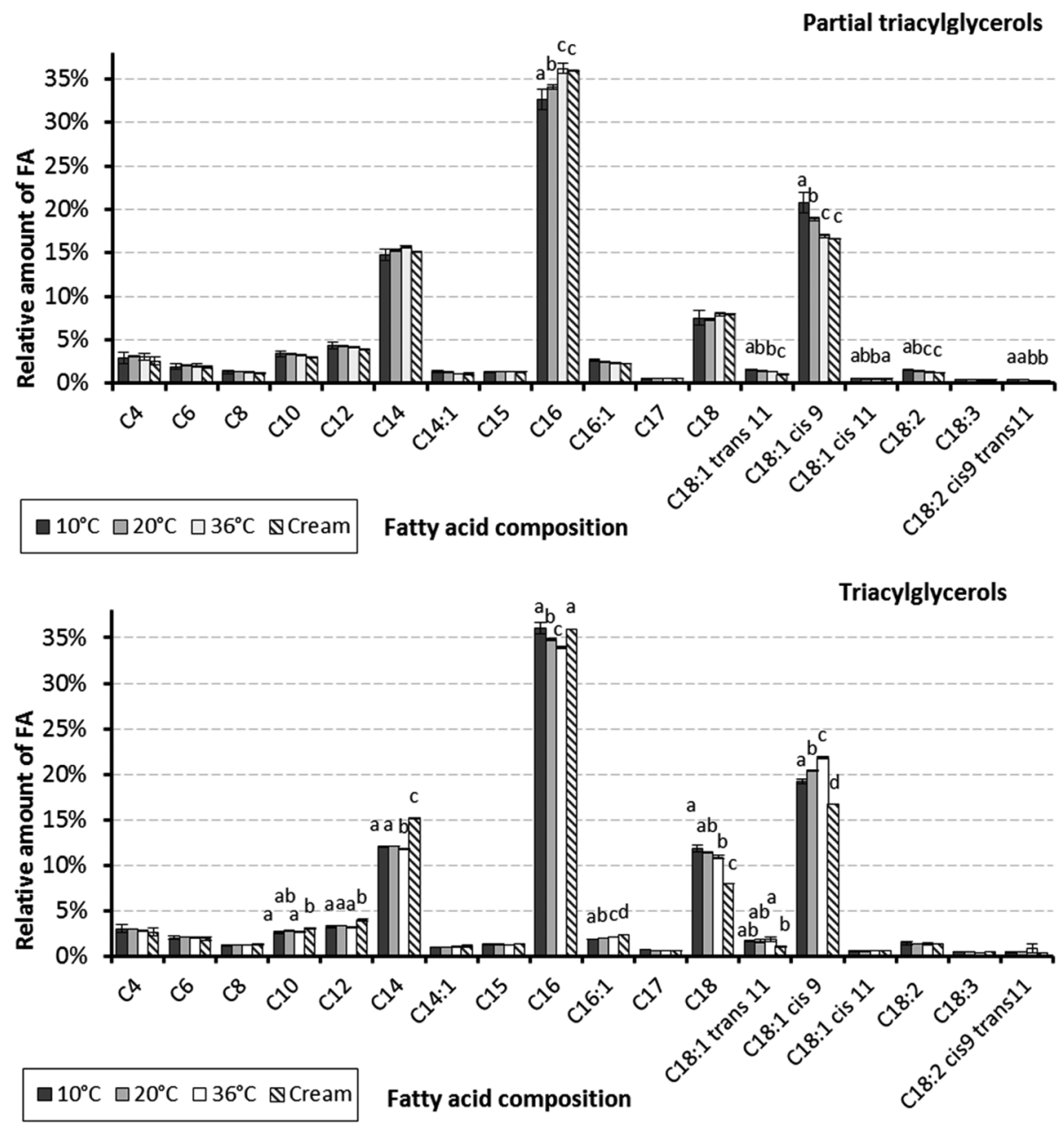

Fig. 3. The main fatty acid composition of lipid classes after lipolysis at different temperatures. Each point represents the average of three determinations on separate milk samples \pm standard deviation. Values with the same letter superscripts are not significantly different (only differences are marked on the figure; $P<0.05$; means were compared using Fisher's least significance difference $(L S D)$ test) 
the released fatty acids varied depending on the incubation temperature of the studied sample. These differences are visible in the contents of the main fatty acid fractions. For partial triacylglycerols, the proportion of C16 saturated acid increased with increasing incubation temperature. The content of $\mathrm{C} 18: 1$ cis 9 acid in this fraction decreased with increasing temperature. For the fraction of triacylglycerols, these changes proceeded similarly, but in the opposite direction and were even more clearly marked. There was a decrease in the content of C14, C16 and C18 saturated acids, while with increasing temperature there was an increase in the content of oleic acid (C18:1 cis 9) and palmitoleic acid C16:1 in this fraction. Similar trends may also have occurred for other fatty acids. However, their low content makes it impossible or difficult to detect them. The partial triacylglycerols are the fraction which was hydrolysed under the effect of lipase. Therefore, at lower temperatures, the fractions hydrolysed to a greater degree were those which contained more unsaturated oleic acid, but contained less C14, C16 and C18 saturated acids. Since triacylglycerols are the fraction which was not hydrolysed under the effect of lipase, for these fatty acids the opposite is true. The obtained results indicate that, depending on the temperature, not only a different amount, but also different fractions of milk fat were hydrolysed under the effect of lipase.

\section{DISCUSSION}

Studies indicating that a significant role is played by the structure and physical state of fat emulsion on its bioavailability under the effect of lipase are limited. Bonnaire et al. (2008) demonstrated differences in the amount of fatty acids released under the effect of lipase for fat droplets in emulsion in a liquid and solid state, but they did not determine the composition of the released fatty acids, only their overall amount. The results obtained in this study indicate that, depending on the temperature, not only a different amount, but also different fractions of milk fat were hydrolysed by lipase.

One factor which has an effect on the digestion of milk fat is the structure of the interface, which modifies substrate availability for lipolysis (Berton et al., 2012; Smoczyński et al., 2012). This structure may occur in various forms at various temperatures, depending on its composition. Phospholipids differ in their structures and in the contents of fatty acids, which may contribute to differences in the membrane's properties, depending on the temperature. It is known that there are domains with a different structure in the milk fat globule membrane known as "lipid rafts" (Lopez et al., 2010), whose properties may change depending on temperature and, thus, have an effect on the access of lipase to the fat substrate inside a milk fat globule.

The physical state of the substrate may have an effect on the bioavailability of milk fat for lipase. Since milk fat is a very complex mixture of triacylglycerols, different fractions may exhibit different physical properties depending on the temperature. Bonnaire et al. (2008) demonstrated that lipase was able to hydrolyse fat in emulsion occurring in a solid form, but to a lesser extent. In the solid state, hydrocarbon chains may be more closely packed and less readily available as a substrate for the hydrolysis reaction. The $\beta$ form of fat crystals has a particularly closely-packed structure (Lopez et al., 2001). At the same time, while crystals of triacylglycerols are being formed, their internal arrangement in a globule may change. It was demonstrated that certain fractions of triacylglycerols crystallise separately, independently of other fractions (Lopez et al., 2001). Crystallisation requires a precise arrangement of the smallest structural elements forming the crystal. The correct arrangement enables them to take the correct positions in the crystal structure and to form a network of bonds. Thus, the separation of homogeneous triacylglycerol fractions from other fractions may enable the formation of crystal structures inside a milk fat globule. Some of the fractions may then be hydrolysed, while other fractions are not hydrolysed, which seems to be confirmed by the results in this study.

The digestion of triacylglycerols takes place at physiological temperature, but consumed milk fat may differ in temperature. The performed study indicates that the temperature of milk fat may have an effect not only on the rate at which fatty acids are released, but also on their profile. It is known that fat is one of the main components of food, which has an effect on satiety regulation. Thus, the differences in released fatty acids at the initial stage of digestion may have a different effect on the mechanisms regulating the sense of satiety, thus modifying the continued process of 
digestion and of absorption of fat in the digestive tract. During digestion of milk fat, lipase occurring in the oral cavity preferentially releases short- and mediumchain fatty acids from the sn1 position. This process is continued in the stomach, resulting in differences in absorption, depending on the length of a chain. These differences may be intensified, depending on the milk temperature.

\section{CONCLUSIONS}

In this work, lipolysis occurred at all three temperatures in the studied milk samples, but the differences were found not only in the amount, but also in the composition of fatty acids released. Therefore, the obtained results may reflect the differences in the rate of lipolysis for different triacylglycerol fractions. For the fractions in a liquid state, the lipolysis rate was faster than for the fractions in a solid state. This may partly result from more intense flocculation of fat globules, which may accelerate lipolysis. The differences in the state of matter of these fractions result from their composition and the content of various fatty acids. Acids containing unsaturated bonds (and acids with a shorter hydrocarbon chain) have an effect on higher liquidity of the triacylglycerol fraction and these were preferentially released under the influence of lipase at lower temperatures. The obtained results also highlight the possibility of profiling the hydrolysis of milk fat when different temperatures are used.

\section{REFERENCES}

Bauer, E., Jakob, S., Mosenthin, R. (2005). Principles of physiology of lipid digestion. Asian-Australas. J. Anim. Sci., 18, 282-295. https://doi.org/10.5713/ajas.2005.282

Berton, A., Rouvellac, S., Robert, B., Rousseau, F., Lopez, C., Crenon, I. (2012). Effect of the size and interface composition of milk fat globules on their in vitro digestion by the human pancreatic lipase: Native versus homogenized milk fat globules. Food Hydrocoll., 29, 123134. http://dx.doi.org/10.1016/j.foodhyd.2012.02.016

Bitman, J., Wood, D. I. (1990). Changes in milk fat phospholipids during lactation. J. Dairy Sci., 73, 1208-1216. http://dx.doi.org/10.3168/jds.S0022-0302(90)78784-X

Blackberg, L., Hernell, O., Olivecrona, T. (1981). Hydrolysis of human milk fat globules by pancreatic lipase. Role of colipase, phospholipase A2 and bile salts. J. Clin. Invest., 67, 1748-1752.

Bonnaire, L., Sandra, S., Helgason, T., Decker, E. A., Weiss, J., McClements, D. J. (2008). Influence of lipid physical state on the in vitro digestibility of emulsified lipids. J. Agr. Food Chem., 56, 3791-3797. https://dx.doi. org/10.1021/jf800159e

Deeth, C. H., Fitz-Gerald, H. C., Wood, A. F. (1975). Convenient method for determining the extend of lipolysis in milk. Aust. J. Dairy Technol., 9, 109-111.

Hamilton, J. G., Comai, K. (1988). Rapid separation of neutral lipids, free fatty acids and polar lipids using prepacked silica sep-pak columns. Lipids, 23, 1146-1149.

Hara, A., Radin, N. S. (1978). Lipid extraction of tissues with a low-toxicity solvent. Anal. Biochem., 90, 420-426.

ISO-IDF (2002). Milk fat-preparation of FA methyl esters. International Standard ISO 15884-IDF 182: 2002.

Lopez, C., Lesieur, P., Bourgaux, C., Keller, G., Ollivon, M. (2001). Thermal and structural behavior of milk fat. 2 . Crystalline forms obtained by slow cooling of cream. J. Colloid Interf. Sci., 240. 150-161.

Lopez, C., Madec, M. N., Jimenez-Flores, R. (2010). Lipid rafts in the bovine milk fat globule membrane revealed by the lateral segregation of phospholipids and heterogeneous distribution of glycoproteins. Food Chem., 120, 22 33. http://dx.doi.org/10.1016/j.foodchem.2009.09.065

MacGibbon, A. H. K., Taylor, M. W. (2006). Composition and structure of bovine milk lipids. In: P. F. Fox, P. L. H. McSweeney (Eds), Advanced dairy chemistry (pp. 1-42). New York: Springer.

Mather, I. H. (2000). A review and proposed nomenclature for major proteins of the milk-fat globule membrane. J. Dairy Sci., 83, 203-247. http://dx.doi.org/10.3168/jds. S0022-0302(00)74870-3

Parodi, P. W. (2004). Milk fat in human nutrition. Aust. J. Dairy Technol., 59, 3-59.

Smoczyński, M., Kiełczewska, K. (2014). Fractal and physico-chemical analysis of cows' milk fat globules after lipolysis. J. Food Nutr. Res., 53, 207-216.

Smoczyński, M., Staniewski, B., Kiełczewska, K. (2012). Composition and structure of the bovine milk globule membrane - some nutritional and technological implications. Food Rev. Int., 28, 188-202. http://dx.doi.org/10.1 080/87559129.2011.595024

United States Pharmacopeia and National Formulary. (2003). United States Pharmacopeial Convention, Rockville, MD, USA. 